\title{
SENSITIVITY ANALYSIS OF THE CRYSTALLIZATION MODEL WITH RESPECT TO COOLING CONDITIONS
}

\author{
Romuald Szopa ${ }^{1}$, Wioletta Tuzikiewicz ${ }^{2}$, Jaroslaw Siedlecki ${ }^{3}$ \\ ${ }^{I}$ Faculty of Civil Engineering, Czestochowa University of Technology \\ Częstochowa, Poland \\ ${ }^{2,3}$ Institute of Mathematics, Czestochowa University of Technology \\ Częstochowa, Poland \\ Iszopa@word.katowice.pl, ${ }^{2}$ wioletta.tuzikiewicz@im.pcz.pl, ${ }^{3}$ jaroslaw.siedlecki@im.pcz.pl
}

\begin{abstract}
In the paper the pure metal crystallization process is considered. The cooling conditions of the metal domain are determined by the mould dimensions and thermophysical parameters of the mould material (volumetric specific heat and thermal conductivity). The mathematical model of heat transfer processes proceeding in the system metal - mould is created by the system of heat diffusion partial differential equations corresponding to the sub-domains considered supplemented by the adequate geometrical, physical and boundary-initial conditions. The sensitivity model determining the perturbations of thermal processes due to the perturbations of cooling processes can be constructed using the direct approach (differentiation with respect to parameters analyzed) or using the differential quotients. Both approaches will be discussed in the paper presented.
\end{abstract}

Keywords: moving boundary problems, crystallization of pure metals, sensitivity analysis, numerical methods

\section{Introduction}

The models of thermal processes proceeding in domain of solidifying metal can be divided in to three groups: macro models, micro-macro models and micro ones [1-3]. In this paper the micro-macro approach is discussed. Such a model bases on the well-known Fourier equation with additional term controlling the crystallization process. At the stage of this source function construction the thermal processes proceeding in the micro scale are taken into account. A capacity of source function is proportional to the crystallization rate, more precisely, to time derivative of function $f_{S}(x, t)$ corresponding to the local and temporary volumetric fraction of solid state at the point considered [3-6] and the kinetics of nucleation and nuclei growth determines the local and temporary value of this function. It is assumed that the driving force of nucleation and nuclei growth is the undercooling below the solidification point $T^{*}$.

The sensitivity model determining the changes of the transient temperature field due to the perturbations of parameters appearing in the energy equations and boundary-initial conditions can be found by the differentiation of successive equa- 
tions and conditions with respect to parameter considered (direct approach [7-9]). In the case of micro-macro models (mainly due to the complicated form of source function) the equations creating the sensitivity model are very complex both at the stage of mathematical manipulations and also at the stage of numerical modeling. In this place the simpler approach to the sensitivity functions modeling can be taken into account. The local and temporary values of the sensitivity function defined as a partial derivative of temperature with respect to parameter considered are found using the differential quotients. The boundary initial problem describing the process must be solved twice, the first solution corresponds to the basic input data (set of parameters $p_{1}, p_{2}, p_{k}, \ldots p_{n}$ ), the second one corresponds to the input data containing the disturbed parameter $p_{k}+\Delta p_{k}$. Next, the temporary local values of the sensitivity function $\left(\Delta T / \Delta p_{k}\right)$ can be found.

It should be pointed out that the name 'crystallization' used in this paper corresponds to the metal solidification analyzed in the micro scale.

\section{Mathematical model of the process}

The equation describing the solidification process is of the form

$$
x \in \Omega: \quad c(T) \frac{\partial T(x, t)}{\partial t}=\nabla[\lambda(T) \nabla T(x, t)]+L \frac{\partial f_{S}(x, t)}{\partial t}
$$

where $c(T)$ is a volumetric specific heat of material, $\lambda(T)$ is a thermal conductivity, $L$ is a volumetric latent heat, $T=T(x, t), f_{S}=f_{S}(x, t)$ denote the temperature and the local volumetric solid state fraction.

Now, the following function should be introduced

$$
\omega(x, t)=N(x, t) V(x, t)
$$

where $N$ is a nuclei density [nuclei $/ \mathrm{m}^{3}$ ],$V$ is a single grain volume. The formula (2) is more complicated in the case of different grain volumes and then the integral form determining $\omega(x, t)$ should be introduced.

The most simple model of pure metal crystallization is based on the assumption

$$
f_{S}(x, t)=\omega(x, t)
$$

and then

$$
\frac{\partial f_{S}(x, t)}{\partial t}=\frac{\partial \omega(x, t)}{\partial t}
$$

This so-called 'linear model' [4] determines the geometrical volume (volume fraction) and it is the correct assumption at the first stages of crystallization. To take into account the geometrical limitations of growth in the final stages of the process (collisions between growing grains) the following modification of equation (4) is introduced by Mochnacki and Szopa $[10,11]$ 


$$
\frac{\partial f_{S}(x, t)}{\partial t}=\frac{\partial \omega(x, t)}{\partial t}\left[1-f_{S}(x, t)\right]^{n}
$$

where $n \geq 0$.

The last equation can be written in the form

$$
\frac{\mathrm{d} f_{S}(\omega)}{\left[1-f_{S}(\omega)\right]^{n}}=\mathrm{d} \omega
$$

and the solution fulfilling the condition $\omega=0: f_{S}=0$ is the following:

$$
f_{S}(\omega)=1-[(n-1) \omega+1]^{\frac{1}{1-n}}
$$

One can see that for $n=0$ one obtains the linear model, while for $n=1$ one has

$$
\lim _{n \rightarrow 1} 1-[(n-1) \omega+1]^{\frac{1}{1-n}}=1-\exp (-\omega)
$$

The formula (8) corresponds to the well-known Kolmogoroff exponential model $[2,3,5]$.

Summing up, the source term in energy equation (1) is equal to

$$
L \frac{\partial f_{S}(x, t)}{\partial t}=L \frac{\mathrm{d} f_{S}(\omega)}{\mathrm{d} \omega} \frac{\partial \omega(x, t)}{\partial t}=L[(n-1) \omega+1]^{\frac{n}{1-n}} \frac{\partial \omega(x, t)}{\partial t}
$$

Equation (1) corresponding to the metal domain should be supplemented by the similar equation determining the thermal processes in a mould sub-domain, this means

$$
x \in \Omega_{m}: \quad c_{m}(T) \frac{\partial T_{m}(x, t)}{\partial t}=\nabla\left[\lambda_{m}(T) \nabla T_{m}(x, t)\right]
$$

where $c_{m}$ and $\lambda_{m}$ denote the mould volumetric specific heat and mould thermal conductivity (the considerations presented below concern the constant values of $c_{m}$ and $\left.\lambda_{m}\right)$.

On the external surface of the system the boundary condition in a general form

$$
\Phi\left[T_{m}(x, t), \frac{\partial T_{m}(x, t)}{\partial n}\right]=0
$$

is assumed ( $\partial / \partial n$ denotes a normal derivative). 
On the contact surface between metal and mould the continuity condition is given

$$
x \in \Gamma_{m}:\left\{\begin{array}{l}
-\lambda \frac{\partial T(x, t)}{\partial n}=-\lambda_{m} \frac{\partial T_{m}(x, t)}{\partial n} \\
T(x, t)=T_{m}(x, t)
\end{array}\right.
$$

The initial temperatures for $t=0$ are also known

$$
t=0: \quad T(x, 0)=T_{0}, \quad T_{m}(x, 0)=T_{m 0}
$$

where $T_{0}$ is the pouring temperature, $T_{m 0}$ is the mould initial temperature.

\section{Sensitivity analysis with respect to the cooling conditions}

At first, the sensitivity model concerning the mould thermal conductivity will be discussed. Using the direct version, one should differentiate the basic model with respect to $\lambda_{m}$ meaning

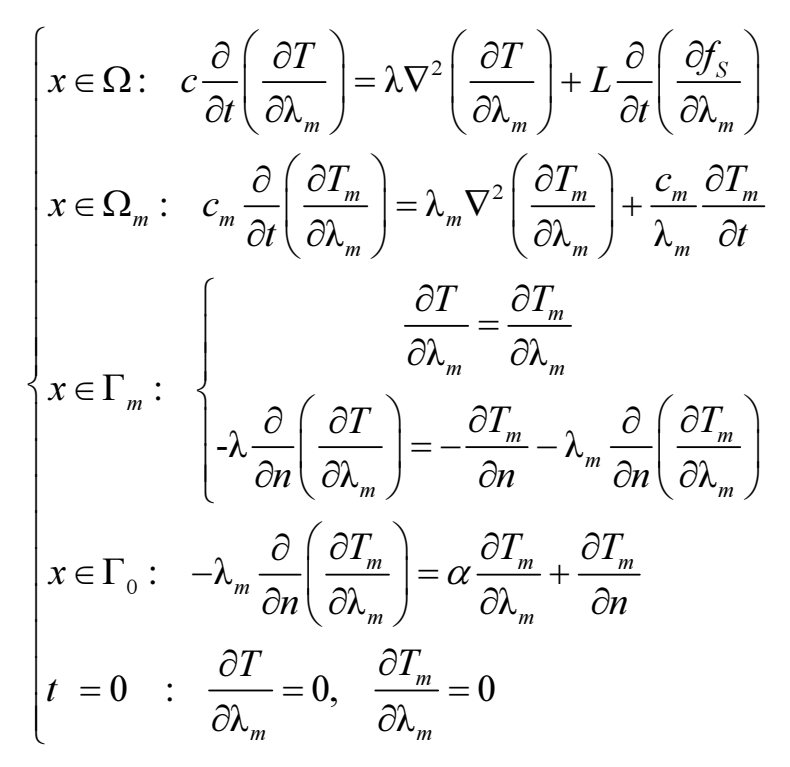

The model (14) was created under the assumption that the thermophysical parameters of metal domain are the constant (taking into account the small interval of temperatures in which the crystallization process occurs this assumption is quite acceptable). Additionally, on the external surface of the mould the Robin condition is introduced ( $\alpha$ is the heat transfer coefficient). Denoting $U=\partial T / \partial \lambda_{m}$ and $U_{m}=\partial T_{m} / \partial \lambda_{m}$ one obtains the following model of sensitivity with respect to the mould thermal conductivity 


$$
\begin{cases}x \in \Omega: & c \frac{\partial U}{\partial t}=\lambda \nabla^{2} U+Q \\
x \in \Omega_{m}: & c_{m} \frac{\partial U_{m}}{\partial t}=\lambda_{m} \nabla^{2} U_{m}+\frac{c_{m}}{\lambda_{m}} \frac{\partial T_{m}}{\partial t} \\
x \in \Gamma_{m}: & \left\{\begin{array}{l}
U=U_{m} \\
-\lambda \frac{\partial U}{\partial n}=-\frac{\partial T_{m}}{\partial n}-\lambda_{m} \frac{\partial U_{m}}{\partial n}
\end{array}\right. \\
x \in \Gamma_{0}: & -\lambda_{m} \frac{\partial U_{m}}{\partial n}=\alpha U_{m}+\frac{\partial T_{m}}{\partial n} \\
t=0 \quad: \quad & U=0, \quad U_{m}=0\end{cases}
$$

Now, the problem of source function $Q$ in the sensitivity equation for metal domain will be explained. As previously mentioned, the driving force of nucleation and nuclei growth is an undercooling below solidification point $T^{*}$. According to the literature, e.g. [2] one can assume that a local and temporary number of nuclei (nuclei density) is proportional to the second power of undercooling below the temperature $T^{*}$

$$
N(x, t)=\eta \Delta T(x, t)^{2}=\eta\left[T^{*}-T(x, t)\right]^{2}
$$

The nucleation stops when $\Delta T(x, t+\Delta t)<\Delta T(x, t)$, additionally for $T(x, t)>T^{*}$ : $N(x, t)=0$ - see: Figure 1 [6], at the same time $\eta$ is the nucleation coefficient.

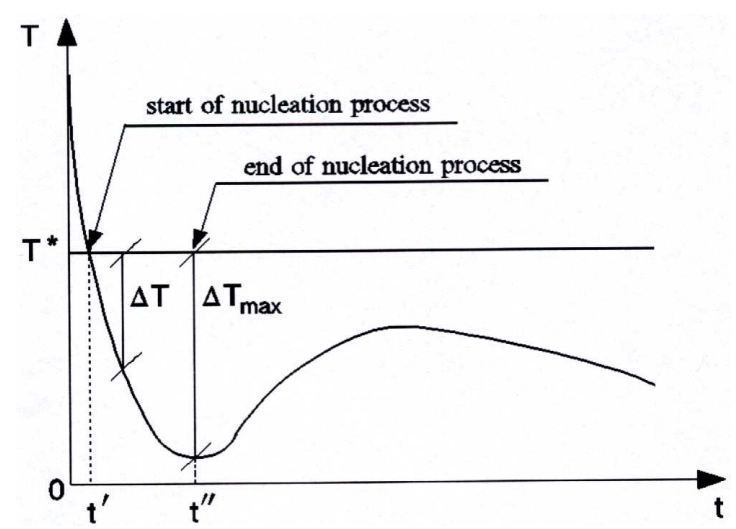

Fig. 1. Temperature history at the point $x$ from metal domain

The rate of solid phase growth (equiaxial grains) is determined by

$$
\frac{\mathrm{d} R(x, t)}{\mathrm{d} t}=\mu \Delta T(x, t)^{m}
$$

where $\mu$ is the growth coefficient, $R$ is grain radius, $m \in[1,2]$. 
Below, the very popular case this means the Kolmogoroff model is considered $(n=1)$. Then the source function (9) in equation (1) can be written as follows:

$$
L \frac{\partial f_{S}}{\partial t}=4 \pi N L \mu \Delta T^{m}\left(\int_{0}^{t} \mu \Delta T^{m} \mathrm{~d} \tau\right)^{2} \exp \left[-\frac{4}{3} \pi N\left(\int_{0}^{t} \mu \Delta T^{m} \mathrm{~d} \tau\right)^{3}\right]
$$

Function (18) should be differentiated with respect to the parameter considered (e.g. mould thermal conductivity). Let us introduce the components

$$
\begin{aligned}
& F_{1}=4 \pi N L \mu \Delta T^{m} \\
& F_{2}=\left(\int_{0}^{t} \mu \Delta T^{m} \mathrm{~d} \tau\right)^{2}
\end{aligned}
$$

and

$$
F_{3}=\exp \left[-\frac{4}{3} \pi N v\left(\int_{0}^{t} \mu \Delta T^{m} \mathrm{~d} \tau\right)^{3}\right]
$$

Derivatives of the above components with respect to mould thermal conductivity are equal to

$$
\begin{gathered}
\frac{\partial F_{1}}{\partial \lambda_{m}}=-4 \pi N L m T^{m-1} U \\
\frac{\partial F_{2}}{\partial \lambda_{m}}=-2 \int_{0}^{t} \mu \Delta T^{m} \mathrm{~d} \tau \int_{0}^{t} \mu m \Delta T^{m-1} U \mathrm{~d} \tau=-2 r_{S} \rho_{S}
\end{gathered}
$$

where

$$
r_{S}=\int_{0}^{t} \mu \Delta T^{m} \mathrm{~d} \tau, \quad \rho_{S}=\int_{0}^{t} \mu m \Delta T^{m-1} U \mathrm{~d} \tau
$$

Additionally

$$
\frac{\partial F_{3}}{\partial p_{i}}=\exp \left[-\frac{4}{3} \pi N\left(\int_{0}^{t} \mu \Delta T^{m} \mathrm{~d} \tau\right)^{3}\right] 4 \pi N\left(\int_{0}^{t} \mu \Delta T^{m} \mathrm{~d} \tau\right)^{2} \int_{0}^{t} \mu m \Delta T^{m-1} U \mathrm{~d} \tau
$$

or

$$
\frac{\partial F_{3}}{\partial p_{i}}=\exp \left[-\frac{4}{3} \pi N r_{S}^{3}\right] 4 \pi N r_{S}^{2} \rho_{S}
$$


Using the formula concerning the product of three components one obtains

$$
\begin{gathered}
Q=4 \pi N L \exp \left[-\frac{4}{3} \pi N r_{S}^{3}\right] . \\
{\left[4 \pi N \mu \Delta T^{m} \rho_{S} r_{S}^{4}-2 \mu \Delta T^{m} \rho_{S} r_{S}-\mu m \Delta T^{m-1} U r_{S}^{2}\right]}
\end{gathered}
$$

The last formula supplements the sensitivity model (15). From the numerical point of view, the numerical solution of the problem is not simple but possible. The essentially simpler approach consists of the application of differential quotients in the place of sensitivity functions. This method requires the knowledge of two basic solutions corresponding to $\lambda_{m}$ and $\lambda_{m}+\Delta \lambda_{m}$.

\section{Example of computations}

Let us consider the aluminium plate (thickness $G=2 \mathrm{~cm}, 1 \mathrm{D}$ task) produced in a typical sand mould. Parameters of metal: $\lambda=150 \mathrm{~W} / \mathrm{mK}, c=3 \cdot 10^{6} \mathrm{~J} / \mathrm{m}^{3} \mathrm{~K}$, $L=9.75 \cdot 10^{8} \mathrm{~J} / \mathrm{m}^{3}, T^{*}=660^{\circ} \mathrm{C}$, parameters of mould: $\lambda_{m}=1.25 \mathrm{~W} / \mathrm{mK}, c_{m}=$ $=1.6 \cdot 10^{6} \mathrm{~J} / \mathrm{m}^{3} \mathrm{~K}$. The constant number of nuclei $N=5 \cdot 10^{10}$ nucl. $/ \mathrm{m}^{3}$ was assumed, growth coefficient $\mu=3 \cdot 10^{-6} \mathrm{~m} / \mathrm{sK}$, initial temperatures $T_{0}=690^{\circ} \mathrm{C}$ and $T_{m 0}=30^{\circ} \mathrm{C}$, correspondingly. In Figures 2 and 3 the curves of sensitivity $U$ for points $x_{1}=1.1 \mathrm{~cm}$ (metal domain) and $x_{2}=2.9, x_{3}=4.4, x_{4}=5.9$ (mould) are shown. The result on the left-hand side corresponds to the solution based on the sensitivity model (15), while the result on the right-hand side corresponds to the solution obtained using the differential quotients, One can see that both solutions are practically the same. It should be pointed out that the basic problem and the sensitivity problem have been solved using the explicit scheme of finite difference method [12].
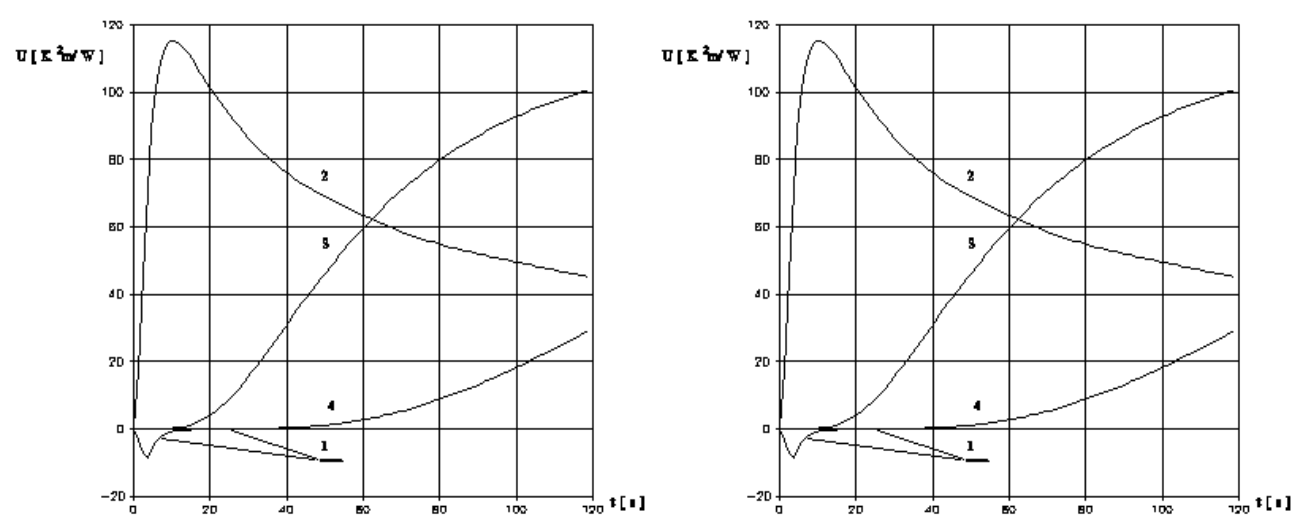

Fig. 2. Course of sensitivity function 


\section{References}

[1] Fraś E., Kapturkiewicz W., Lopez H.F., Macro and micro modelling of the solidification kinetics of casting, AFS Transactions 1993, 92-48, 583-591.

[2] Fraś E., Crystallization of Metals and Alloys, PWN, Warsaw 1992.

[3] Kapturkiewicz W., Modelling of Cast Iron Solidification, AKAPIT, Cracow 2003.

[4] Majchrzak E., Suchy J.S., Szopa R., Linear model of crystallization. Identification of nuclei density, Giessereiforschung, International Foundry Research 2006, 2, 29-32.

[5] Majchrzak E., Piasecka A., Numerical micro/macro model of solidification process, Journal of Materials Processing Technology 1997, 64, 367-276.

[6] Mochnacki B., Computational simulations and applications, [in:] Jianping Zhu (ed.), Numerical Modeling of Solidification Process, INTECH, 2011, 513-542.

[7] Kleiber M., Parameter Sensitivity in Nonlinear Mechanics, J. Wiley \& Sons Ltd., Chichester, England, 1997.

[8] Mochnacki B., Szopa R., Application of sensitivity analysis in numerical simulation of solidification process, [in:] J. Szajnara (ed.), Progress of Foundry Theory and Practice, Polish Ac. of Sciences, Foundry Commission, 2009, 271-286.

[9] Mochnacki B, Majchrzak E., Identification of macro and micro parameters in solidification model, Bulletin of the Polish Academy of Sciences, Technical Sciences 2007, 55, 1, 107-113.

[10] Mochnacki B., Szopa R., Model of pure metal solidification using the power-type function, Journal of Achievements in Materials and Manufacturing Engineering 2007, 22, 65-71.

[11] Mochnacki B., Szopa R., Generalized micro/macro model of crystallization and its numerical realization, International Journal for Multiscale Computational Engineering 2010, 8(3), 259-266.

[12] Mochnacki B., Suchy J.S., Numerical Methods in Computations of Foundry Processes, PFTA, Cracow 1995. 